\title{
RADIAL SYMMETRY OF $n$-MODE POSITIVE SOLUTIONS FOR SEMILINEAR ELLIPTIC EQUATIONS IN A DISC AND ITS APPLICATION TO THE HÉNON EQUATION
}

\author{
NaOKi Shioji — Kohtaro Watanabe
}

Abstract. Let $f \in C((0,1) \times(0, \infty), \mathbb{R})$ and $n \in \mathbb{N}$ with $n \geq 2$ such that for each $u \in(0, \infty), r \mapsto r^{2-2 n} f(r, u):(0,1) \rightarrow \mathbb{R}$ is nonincreasing and let $D=\left\{x=\left(x_{1}, x_{2}\right) \in \mathbb{R}^{2}:|x|<1\right\}$. We show that each positive solution of

$$
\Delta u+f(|x|, u)=0 \quad \text { in } D, \quad u=0 \quad \text { on } \partial D
$$

which satisfies $u(r, \theta)=u(r, \theta+2 \pi / n)$ by the polar coordinates is radially symmetric and $u_{r}(|x|)<0$ for each $r=|x| \in(0,1)$. We apply our result to the Hénon equation.

\section{Introduction}

The radial symmetry of positive solutions of the problem

$$
\begin{cases}\Delta u+f(|x|, u)=0 & \text { in } B, \\ u=0 & \text { on } \partial B,\end{cases}
$$

has been studied by many researchers, where $B$ is the open unit ball in $\mathbb{R}^{N}$ $(N \geq 2)$ and $f \in C((0,1) \times(0, \infty), \mathbb{R})$. Gidas, Ni and Nirenberg [12] showed that if $f$ satisfies that for each $u \in(0, \infty), r \mapsto f(r, u):(0,1) \rightarrow \mathbb{R}$ is nonincreasing, then

2010 Mathematics Subject Classification. 35B06, 35B09, 35J15.

Key words and phrases. Elliptic equations, positive solutions, radial symmetry, Riemannian isometric, the Hénon equation.

The work of the first named author is partially supported by the Grant-in-Aid for Scientific Research (C) (No. 21540214) from Japan Society for the Promotion of Science. 
any $C^{2}(\bar{B})$-positive solution is radially symmetric. For related results, we refer to [1], [4]-[10], [12], [13], [17]-[20], [22]-[27], [29], [30]. For recent developments of the symmetry of positive solutions of (1.1), see [2], [3], [15], [28], [33] and references therein.

In this paper, we give a radial symmetry result for an $n$-mode positive solution of

$$
\begin{cases}\Delta u+f(|x|, u)=0 & \text { in } D \backslash\{0\}, \\ u=0 & \text { on } \partial D,\end{cases}
$$

where $D=\left\{x=\left(x_{1}, x_{2}\right) \in \mathbb{R}^{2}:|x|<1\right\}$. We say a function $u: \bar{D} \rightarrow \mathbb{R}$ is $n$-mode with $n \in \mathbb{N}$ if it is $2 \pi / n$ rotationally invariant, i.e.

$$
u(r, \theta)=u\left(r, \theta+\frac{2 \pi}{n}\right) \quad \text { for each }(r, \theta) \in[0,1] \times \mathbb{R}
$$

by the polar coordinates. We say $u$ is of class $C^{n}(n \in \mathbb{N})$ at the origin if $u$ is of class $C^{n-1}$ in a neighbourhood of the origin and each $(n-1)$-th partial derivative is totally differentiable at the origin.

Now, we show our first result.

THEOREM 1.1. Let $n \in \mathbb{N}$ with $n \geq 2$ and $f \in C((0,1) \times(0, \infty), \mathbb{R})$ such that:

(a) for each $u \in(0, \infty), r \mapsto r^{2-2 n} f(r, u):(0,1) \rightarrow \mathbb{R}$ is nonincreasing,

(b) for each $r_{0} \in(0,1)$ and $M \in(0, \infty)$,

$\sup \left\{\left|\frac{f\left(r, u_{1}\right)-f\left(r, u_{2}\right)}{u_{1}-u_{2}}\right|:\left(r, u_{1}, u_{2}\right) \in\left(r_{0}, 1\right) \times(0, M]^{2}, u_{1} \neq u_{2}\right\}<\infty$.

Let $u \in C^{2}(D \backslash\{0\}) \cap C(\bar{D})$ be an n-mode positive solution of (1.2). Assume that $u$ is of class $C^{n}$ at the origin or $u(0)>u(x)$ for all $x \in \bar{D} \backslash\{0\}$. Then $u$ is radially symmetric and $u_{r}(|x|)<0$ for $r=|x| \in(0,1)$.

REMARK 1.2. The case $n=1$ corresponds to Gidas-Ni-Nirenberg's theorem.

Under the condition $u(x) \rightarrow \infty$ as $|x| \rightarrow 0$, we can show the following.

Theorem 1.3. Let $n$ and $f$ be as in Theorem 1.1. Let $u \in C^{2}(D \backslash\{0\}) \cap$ $C(\bar{D} \backslash\{0\})$ be an n-mode positive solution of

$$
\begin{cases}\Delta u+f(|x|, u)=0 & \text { in } D \backslash\{0\}, \\ u=0 & \text { on } \partial D, \\ \lim _{|x| \rightarrow 0} u(x)=\infty . & \end{cases}
$$

Then $u$ is radially symmetric and $u_{r}(|x|)<0$ for $r=|x| \in(0,1)$. 
As an application, we consider the Hénon equation

$$
\begin{cases}\Delta u+|x|^{\alpha}|u|^{p-2} u=0 & \text { in } D, \\ u=0 & \text { on } \partial D\end{cases}
$$

with $\alpha \in(0, \infty)$ and $p \in(2, \infty)$. Smets, Willem and Su [32, Theorem 6] showed that the problem has a nonradial positive solution for sufficiently large $\alpha>0$ (including the higher dimensional case). We show that the solution they obtained does not have an $n$-mode symmetry in some sense and that the number of the nonradial positive solutions tends to infinity as $\alpha \rightarrow \infty$.

For each $\alpha>0$, we denote by $\lceil\alpha\rceil$ and $\lfloor\alpha\rfloor$ the smallest integer greater than or equal to $\alpha$ and the largest integer less than or equal to $\alpha$, respectively. For each $\alpha \geq 0$ and $p>2$, we set

$$
R_{\alpha, p}(u)=\frac{\int_{D}|\nabla u|^{2} d x}{\left(\int_{D}|x|^{\alpha}|u|^{p} d x\right)^{2 / p}} \text { for each } u \in H_{0}^{1}(D) \backslash\{0\} .
$$

THEOREM 1.4. There hold the following:

(a) If $\alpha \in(0, \infty), p \in(2, \infty)$ and $u$ is an $n$-mode, positive solution of $(1.4)$ with $n \geq 1+\lceil\alpha / 2\rceil$, then $u$ is radially symmetric.

(b) For each $\alpha, p \in(2, \infty)$, if $n_{\alpha} \geq 1$ then problem (1.4) has a nonradial, $n$-mode positive solution $u_{n}$ for $n=1, \ldots, n_{\alpha}$ such that

$$
R_{\alpha, p}\left(u_{1}\right)<\ldots<R_{\alpha, p}\left(u_{n_{\alpha}}\right),
$$

where $n_{\alpha}$ is the greatest integer less than

$$
\left(\frac{\alpha+2}{2 \alpha}\right)^{4 /(p-2)}\left(\frac{\alpha-2}{\alpha}\right)^{2 \alpha /(p-2)}\left(1+\frac{\alpha}{2}\right) .
$$

In particular, there hold the following:

(i) For each $\alpha \in(2, \infty)$, if $p \in(2, \infty)$ is large enough, then $n_{\alpha}=\lceil\alpha / 2\rceil$, that is, problem (1.4) has a nonradial, n-mode positive solution for $n=1, \ldots,\lceil\alpha / 2\rceil\left(=n_{\alpha}\right)$ satisfying (1.5).

(ii) For each $p \in(2, \infty)$ and $m \in \mathbb{N}$, if $\alpha \in(2, \infty)$ is large enough, problem (1.4) has a nonradial, $n$-mode positive solution $u_{n}$ for $n=$ $1, \ldots, m$ such that $R_{\alpha, p}\left(u_{1}\right)<\ldots<R_{\alpha, p}\left(u_{m}\right)$. In particular, for each $p \in(2, \infty)$, the number of nonradial positive solutions of (1.4) tends to infinity as $\alpha \rightarrow \infty$.

REMARK 1.5. In the theorem above, we can easily see $n_{\alpha} \leq\lceil\alpha / 2\rceil$.

REMARK 1.6. In the case that $\alpha>0$ is sufficiently small, Kajikiya [16, Theorem 2.4] showed that the least energy solution of (1.4) is radially symmetric. 
We study further the symmetry of $n$-mode positive solutions for (1.1). In some of radial symmetry results for positive solutions of semilinear elliptic equations, geometry plays an important role. Naito, Nishimoto and Suzuki [25] considered the case that $\left(1-r^{2}\right)^{2} f(r, u):(0,1) \rightarrow \mathbb{R}$ is decreasing for each $u \in(0, \infty)$. Using hyperbolic geometry, they showed each positive solution of (1.1) is radially symmetric; see also [26] for the higher dimensional case. The authors [31] studied the case that $a \in(-1,1]$ and $\left(1+a r^{2}\right)^{2} f(r, u):(0,1) \rightarrow \mathbb{R}$ is nonincreasing for each $u \in(0, \infty)$, and they showed that each positive solution (1.1) is radially symmetric. We generalize Theorems 1.1 and 1.3 by using elliptic and hyperbolic geometry. In the following, the case $a=0$ corresponds to Theorem 1.1.

Theorem 1.7. Let $n \in \mathbb{N}$ with $n \geq 2, a \in[-1,1]$ and $f \in C((0,1) \times$ $(0, \infty), \mathbb{R})$ such that

(a) for each $u \in(0, \infty), r \mapsto\left(1+a r^{2 n}\right)^{2} r^{2-2 n} f(r, u):(0,1) \rightarrow \mathbb{R}$ is nonincreasing in the case of $a \in(-1,1]$, and it is decreasing in the case of $a=-1$,

(b) for each $r_{0} \in(0,1)$ and $M \in(0, \infty)$,

$\sup \left\{\left|\frac{f\left(r, u_{1}\right)-f\left(r, u_{2}\right)}{u_{1}-u_{2}}\right|:\left(r, u_{1}, u_{2}\right) \in\left(r_{0}, 1\right) \times(0, M]^{2}, u_{1} \neq u_{2}\right\}<\infty$.

Let $u \in C^{2}(D \backslash\{0\}) \cap C(\bar{D})$ be an n-mode positive solution of (1.2). Assume that $u$ is of class $C^{n}$ at the origin or $u(0)>u(x)$ for all $x \in \bar{D} \backslash\{0\}$. Then $u$ is radially symmetric and $u_{r}(|x|)<0$ for $r=|x| \in(0,1)$.

REMARK 1.8. The case $n=1$ and $a=-1$ corresponds to [25, Theorem 1] (see also [26, Theorem 1]), and the case $n=1$ and $a \in(-1,0) \cup(0,1]$ corresponds to [31, Theorem 1].

As a consequence of Theorem 1.7, we have the following:

Corollary 1.9. Let $n, a$ and $f$ be as in Theorem 1.7. Assume $f(r, u) \geq 0$ for each $(r, u) \in(0,1) \times(0, \infty)$. Let $u \in C^{2}(D \backslash\{0\}) \cap C(\bar{D})$ be a positive solution of (1.2) which is of class $C^{k}(k \in \mathbb{N} \cup\{\infty\})$ at the origin. If $u$ is m-mode with $n \leq m \leq k$, then $u$ is radially symmetric.

In the following, the case $a=0$ corresponds to Theorem 1.3.

Theorem 1.10. Let $n$, $a$ and $f$ be as in Theorem 1.7. Let $u \in C^{2}(D \backslash\{0\}) \cap$ $C(\bar{D} \backslash\{0\})$ be an $n$-mode positive solution of (1.3). Then $u$ is radially symmetric and $u_{r}(|x|)<0$ for $r=|x| \in(0,1)$.

This paper is organized as follows. In Section 2, we give the proofs of Theorems 1.1 and 1.3, and in Section 3, we give the proof of Theorem 1.4. Although 
we need some devices to prove Theorems 1.7 and 1.10 , we can prove them similarly. So we give a proof of Theorem 1.7 in Appendix and we omit the proof of Theorem 1.10.

\section{Proofs of Theorems 1.1 and $\mathbf{1 . 3}$}

First, we give the proof of Theorem 1.1. Let $n, f$ and $u$ be as in Theorem 1.1. By using the polar coordinates, we define $\widetilde{u}: \bar{D} \rightarrow \mathbb{R}$ by $\widetilde{u}(r, \theta)=u\left(r^{1 / n}, \theta / n\right)$ for $(r, \theta) \in \bar{D}$. Since $u$ is $n$-mode and $u$ satisfies $(1.2)$, we can see that $\widetilde{u} \in$ $C^{2}(D \backslash\{0\}) \cap C(\bar{D})$ and $\widetilde{u}$ satisfies

$$
\begin{cases}\Delta \widetilde{u}+\widetilde{f}(|x|, \widetilde{u})=0 & \text { in } D \backslash\{0\} \\ \widetilde{u}=0 & \text { on } \partial D\end{cases}
$$

where $\tilde{f} \in C((0,1) \times(0, \infty), \mathbb{R})$ is given by $\tilde{f}(r, t)=n^{-2} r^{(2-2 n) / n} f\left(r^{1 / n}, t\right)$ for $(r, t) \in(0,1) \times(0, \infty)$, and $\widetilde{f}$ satisfies

$$
\text { for each } t>0, r \mapsto \widetilde{f}(r, t) \text { is nonincreasing. }
$$

Indeed, from

$$
\begin{aligned}
\Delta \widetilde{u}(r, \theta)+\widetilde{f}(r, \widetilde{u}(r, \theta))= & \frac{1}{n^{2}} r^{(2-2 n) / n}\left(u_{r r}\left(r^{1 / n}, \frac{\theta}{n}\right)+\frac{1}{r^{1 / n}} u_{r}\left(r^{1 / n}, \frac{\theta}{n}\right)\right. \\
& \left.+\frac{1}{r^{2 / n}} u_{\theta \theta}\left(r^{1 / n}, \frac{\theta}{n}\right)+f\left(r^{1 / n}, u\left(r^{1 / n}, \frac{\theta}{n}\right)\right)\right)=0,
\end{aligned}
$$

we have (2.1), and we can easily see (2.2).

For each $\lambda \in(0,1)$, we set $\Sigma_{\lambda}=\left\{x \in D: x_{1}>\lambda\right\}$ and we define $h_{\lambda}: \overline{\Sigma_{\lambda}} \rightarrow \bar{D}$ by $h_{\lambda}(x)=\left(2 \lambda-x_{1}, x_{2}\right)$ for $x=\left(x_{1}, x_{2}\right) \in \overline{\Sigma_{\lambda}}$. We note that $h_{\lambda}$ satisfies

$$
\left|h_{\lambda}(x)\right|<|x| \text { for each } \lambda \in(0,1) \text { and } x \in \Sigma_{\lambda} \cup \operatorname{Int}_{\partial D}\left(\overline{\Sigma_{\lambda}} \cap \partial D\right) .
$$

Here, for a subset $E$ of $\partial D$, we denote by $\operatorname{Int}_{\partial D} E$, the interior set of $E$ with respect to the relative topology of $\partial D$. We set $x_{\lambda}=(2 \lambda, 0)$ for $\lambda \in(0,1)$. For the sake of convenience in the arguments in Appendix, we set $\widehat{\lambda}=1 / 2$ in this section. We can see

$$
x_{\lambda} \in \begin{cases}\Sigma_{\lambda} & \text { for each } \lambda \in(0, \widehat{\lambda}), \\ \partial \Sigma_{\lambda} & \text { for } \lambda=\widehat{\lambda} \\ \mathbb{R}^{2} \backslash \overline{\Sigma_{\lambda}} & \text { for each } \lambda \in(\widehat{\lambda}, 1)\end{cases}
$$

and

$$
h_{\lambda}\left(x_{\lambda}\right)=0 \quad \text { for each } \lambda \in(0, \widehat{\lambda}] .
$$

For the sake of completeness, we note that $\Sigma_{\lambda} \backslash\left\{x_{\lambda}\right\}=\Sigma_{\lambda}$ for each $\lambda \in[\widehat{\lambda}, 1)$ and $\overline{\Sigma_{\lambda}} \backslash\left\{x_{\lambda}\right\}=\overline{\Sigma_{\lambda}}$ for each $\lambda \in(\widehat{\lambda}, 1)$. 
We define $v_{\lambda} \in C^{2}\left(\Sigma_{\lambda} \backslash\left\{x_{\lambda}\right\}\right) \cap C\left(\overline{\Sigma_{\lambda}}\right)$ and $c_{\lambda} \in L^{\infty}\left(\Sigma_{\lambda}\right)$ by

$$
v_{\lambda}(x)=\widetilde{u}(x)-\widetilde{u}\left(h_{\lambda}(x)\right) \quad \text { for } x \in \overline{\Sigma_{\lambda}}
$$

and

$$
c_{\lambda}(x)= \begin{cases}-\frac{\widetilde{f}(|x|, \widetilde{u}(x))-\widetilde{f}\left(|x|, \widetilde{u}\left(h_{\lambda}(x)\right)\right)}{\widetilde{u}(x)-\widetilde{u}\left(h_{\lambda}(x)\right)} & \text { for } x \in \Sigma_{\lambda} \text { with } v_{\lambda}(x) \neq 0 \\ 0 & \text { for } x \in \Sigma_{\lambda} \text { with } v_{\lambda}(x)=0\end{cases}
$$

respectively. By the assumptions of Theorem 1.1, we can see

$$
\sup _{r<\lambda<1} \underset{x \in \Sigma_{\lambda}}{\operatorname{ess} \sup }\left|c_{\lambda}(x)\right|<\infty \quad \text { for each } r \in(0,1)
$$

and

$$
-\Delta v_{\lambda}(x)+c_{\lambda}(x) v_{\lambda}(x) \leq 0 \quad \text { for } \lambda \in(0,1) \text { and } x \in \Sigma_{\lambda} \backslash\left\{x_{\lambda}\right\} .
$$

Now, we apply the moving plane argument. Since $\widetilde{u}$ may not be $C^{2}$ at the origin, we need additional arguments. We set

$$
A_{1}=\left\{\lambda \in[\widehat{\lambda}, 1): v_{\lambda}(x)<0 \text { for each } x \in \Sigma_{\lambda}\right\} \quad \text { and } \quad \mu_{1}=\inf _{\lambda \in A_{1}} \lambda .
$$

LEMma 2.1. $A_{1} \neq \emptyset$.

Proof. Let $\lambda \in[\widehat{\lambda}, 1)$ such that $\lambda$ is sufficiently close to 1 . Then we can easily see $v_{\lambda}(x) \leq 0$ for $x \in \partial \Sigma_{\lambda}$ and $v_{\lambda}(x)<0$ for $x \in \operatorname{Int}_{\partial D}\left(\partial D \cap \partial \Sigma_{\lambda}\right)$ from (2.3). Since $\left|\Sigma_{\lambda}\right| \ll 1$ and (2.9) holds, by the Alexandroff-Bakelman-Pucci inequality [14, Theorem 9.1], which is abbreviated to the ABP inequality below, we have $v_{\lambda} \leq 0$ on $\overline{\Sigma_{\lambda}}$. By the strong maximum principle, we have $v_{\lambda}<0$ in $\Sigma_{\lambda}$. Thus we have shown $\lambda \in A_{1}$, which yields $A_{1} \neq \emptyset$.

Lemma 2.2. $\mu_{1}=\widehat{\lambda}$ and $\widehat{\lambda} \in A_{1}$.

Proof. We have $v_{\mu_{1}}(x) \leq 0$ for $x \in \Sigma_{\mu_{1}}$. Since (2.9) holds with $\lambda=\mu_{1}$ and $v_{\mu_{1}}(x)<0$ for $x \in \operatorname{Int}_{\partial D}\left(\partial \Sigma_{\mu_{1}} \cap \partial D\right)$ from (2.3), by the strong maximum principle, we have $v_{\mu_{1}}(x)<0$ for $x \in \Sigma_{\mu_{1}}$, which yields $\mu_{1} \in A_{1}$. So it is enough to show $\mu_{1}=\widehat{\lambda}$. Suppose not, i.e. $\mu_{1}>\widehat{\lambda}$. Let $G$ be an open set such that $\bar{G} \subset \Sigma_{\mu_{1}}$ and $\left|\Sigma_{\mu_{1}} \backslash \bar{G}\right| \ll 1$. We have $\max _{x \in \bar{G}} v_{\mu_{1}}(x)<0$. Let $0<\varepsilon \ll 1$. Then we have $\max _{x \in \bar{G}} v_{\mu_{1}-\varepsilon}(x)<0$ and $\left|\Sigma_{\mu_{1}-\varepsilon} \backslash \bar{G}\right| \ll 1$. Since (2.9) holds with $\lambda=\mu_{1}-\varepsilon, v_{\mu_{1}-\varepsilon}(x) \leq 0$ for $x \in \partial\left(\Sigma_{\mu_{1}-\varepsilon} \backslash \bar{G}\right)$ and $v_{\mu_{1}-\varepsilon}(x)<0$ for $x \in$ $\left(\operatorname{Int}_{\partial D}\left(\partial \Sigma_{\mu_{1}-\varepsilon} \cap \partial D\right)\right) \cup \partial G$, by the ABP inequality and the strong maximum principle, we have $v_{\mu_{1}-\varepsilon}(x)<0$ for $x \in \Sigma_{\mu_{1}-\varepsilon}$, which yields $\mu_{1}-\varepsilon \in A_{1}$. This is a contradiction. Thus we have shown $\mu_{1}=\widehat{\lambda}$ and $\widehat{\lambda} \in A_{1}$.

We set

$$
A_{2}=\left\{\lambda \in(0, \widehat{\lambda}): v_{\lambda}(x)<0 \text { for each } x \in \Sigma_{\lambda}\right\} \quad \text { and } \quad \mu_{2}=\inf _{\lambda \in A_{2}} \lambda .
$$


Lemma 2.3. $A_{2} \neq \emptyset$.

Proof. We note that $x_{\widehat{\lambda}}=(1,0)$. Let $G$ be an open set such that $\bar{G} \subset \Sigma_{\widehat{\lambda}}$ and $\left|\Sigma_{\widehat{\lambda}} \backslash G\right| \ll 1$. From $\widehat{\lambda} \in A_{1}$ and $\bar{G} \subset \Sigma_{\widehat{\lambda}}$, we have $\max _{x \in \bar{G}} v_{\widehat{\lambda}}(x)<0$. Let $\lambda \in(0, \hat{\lambda})$ such that $\lambda$ is sufficiently close to $\widehat{\lambda}$. We note $\left|\Sigma_{\lambda} \backslash \bar{G}\right| \ll 1$ and $x_{\lambda}$ is close to $(1,0)$. We choose a sufficiently small open neighbourhood $U$ of $x_{\lambda}$ with $\bar{U} \subset \Sigma_{\lambda}$, and we set $H=G \cup U$. Then we have $v_{\lambda}(x)<0$ for $x \in \bar{H}$, $v_{\lambda}(x) \leq 0$ for $x \in \partial \Sigma_{\lambda} \cup \partial H$ and $\left|\Sigma_{\lambda} \backslash \bar{H}\right| \ll 1$. Since (2.9) holds on $\Sigma_{\lambda} \backslash \bar{H}$, by the ABP inequality and the strong maximum principle, we have $v_{\lambda}<0$ on $\Sigma_{\lambda}$, which yields $\lambda \in A_{2}$.

Lemma 2.4. Assume that $u$ is of class $C^{n}$ at the origin. Then there holds

$$
\frac{\partial\left(\widetilde{u} \circ h_{\mu_{2}}\right)}{\partial x_{1}}\left(x_{\mu_{2}}\right)=0 .
$$

ProOf. We will show that each $j$-th partial derivative of $u$ at $(0,0)$ is zero for all $j=1, \ldots, n$. Since $u$ is $n$-mode with $n \geq 2$ and $u$ is totally differentiable at the origin, we can easily see $\nabla u(0,0)=(0,0)$. Let $j \in\{1, \ldots, n\}$. By using mathematical induction, we can see that each $(j-1)$-th partial derivative of $u$ is also $n$-mode (in a small open ball whose center is the origin), it is totally differentiable at zero and hence each $j$-th partial differential coefficient of $u$ at the origin is zero. Hence we have shown

$$
\frac{\partial^{j} u}{\partial x_{1}^{k} \partial x_{2}^{j-k}}(0,0)=0 \text { for } j=1, \ldots, n \text { and } k=0, \ldots, j \text {. }
$$

Next, we will show

$$
\lim _{t \rightarrow+0} \frac{u\left(t^{1 / n} \alpha(t), t^{1 / n} \beta(t)\right)-u(0,0)}{t}=0
$$

where $0<\varepsilon \ll 1$ and $\alpha, \beta \in C([0, \varepsilon),[0, \infty))$ are any bounded functions. By Taylor's theorem and (2.13), for each $0<t \ll 1$, there exists $\zeta_{t} \in(0,1)$ such that

$$
\begin{aligned}
& u\left(t \alpha\left(t^{n}\right), t \beta\left(t^{n}\right)\right) \\
& \quad=u(0,0)+\sum_{k=0}^{n-1} \frac{\left(t \alpha\left(t^{n}\right)\right)^{k}\left(t \beta\left(t^{n}\right)\right)^{n-1-k}}{k !(n-1-k) !} \frac{\partial^{n-1} u}{\partial x_{1}^{k} \partial x_{2}^{n-1-k}}\left(\zeta_{t} t \alpha\left(t^{n}\right), \zeta_{t} t \beta\left(t^{n}\right)\right) .
\end{aligned}
$$

Using (2.13) again, we obtain

$$
\begin{aligned}
\lim _{t \rightarrow+0} \frac{u\left(t \alpha\left(t^{n}\right), t \beta\left(t^{n}\right)\right)-u(0,0)}{t^{n}}=\lim _{t \rightarrow+0} \sum_{k=0}^{n-1} \frac{\left(\alpha\left(t^{n}\right)\right)^{k}\left(\beta\left(t^{n}\right)\right)^{n-1-k}}{k !(n-1-k) !} \\
\frac{\frac{\partial^{n-1} u}{\partial x_{1}^{k} \partial x_{2}^{n-1-k}}\left(\zeta_{t} t \alpha\left(t^{n}\right), \zeta_{t} t \beta\left(t^{n}\right)\right)-\frac{\partial^{n-1} u}{\partial x_{1}^{k} \partial x_{2}^{n-1-k}}(0,0)}{t}=0,
\end{aligned}
$$


which yields (2.14). From the definitions of $\widetilde{u}$ and $h_{\mu_{2}}$ and (2.14), we have

$$
\begin{aligned}
& \lim _{t \rightarrow+0} \frac{\widetilde{u}\left(h_{\mu_{2}}\left(x_{\mu_{2}}+t(1,0)\right)\right)-\widetilde{u}\left(h_{\mu_{2}}\left(x_{\mu_{2}}\right)\right)}{t} \\
&=\lim _{t \rightarrow+0} \frac{u\left(t^{1 / n} \cos \pi / n, t^{1 / n} \sin \pi / n\right)-u(0,0)}{t}=0, \\
& \lim _{t \rightarrow+0} \frac{\widetilde{u}\left(h_{\mu_{2}}\left(x_{\mu_{2}}-t(1,0)\right)\right)-\widetilde{u}\left(h_{\mu_{2}}\left(x_{\mu_{2}}\right)\right)}{t}=\lim _{t \rightarrow+0} \frac{u\left(t^{\frac{1}{n}}, 0\right)-u(0,0)}{t}=0 .
\end{aligned}
$$

Hence we have shown (2.12).

LEMMA 2.5. $\mu_{2}=0$.

Proof. Suppose $\mu_{2} \neq 0$. Then we have $\mu_{2} \in(0, \hat{\lambda})$ by Lemma 2.3 , and we can see $v_{\mu_{2}} \leq 0$ on $\overline{\Sigma_{\mu_{2}}}$. We will show $v_{\mu_{2}}<0$ on $\Sigma_{\mu_{2}} \backslash\left\{x_{\mu_{2}}\right\}$. We have $v_{\mu_{2}}(x)<0$ for $x \in \operatorname{Int}_{\partial D}\left(\partial \Sigma_{\mu_{2}} \cap \partial D\right)$ from (2.3). By (2.9) with $\lambda=\mu_{2}$ and the strong maximum principle, we have $v_{\mu_{2}}<0$ on $\Sigma_{\mu_{2}} \backslash\left\{x_{\mu_{2}}\right\}$.

Next, we will show $v_{\mu_{2}}\left(x_{\mu_{2}}\right)<0$. In the case $u(0)>u(x)$ for $x \in \bar{D} \backslash\{0\}$, we can easily see $v_{\mu_{2}}\left(x_{\mu_{2}}\right)<0$. So we consider the case that $u$ is of class $C^{n}$ at the origin. Suppose $v_{\mu_{2}}\left(x_{\mu_{2}}\right)<0$ does not hold, i.e. $v_{\mu_{2}}\left(x_{\mu_{2}}\right)=0$. Let $\nu_{1}=(-1,0)$ and $\nu_{2}=(1,0)$. From (2.12), we have

$$
\begin{aligned}
& \frac{\partial v_{\mu_{2}}}{\partial \nu_{1}}\left(x_{\mu_{2}}\right)=-\frac{\partial \widetilde{u}}{\partial x_{1}}\left(x_{\mu_{2}}\right)+\frac{\partial\left(\widetilde{u} \circ h_{\mu_{2}}\right)}{\partial x_{1}}\left(x_{\mu_{2}}\right)=-\frac{\partial \widetilde{u}}{\partial x_{1}}\left(x_{\mu_{2}}\right), \\
& \frac{\partial v_{\mu_{2}}}{\partial \nu_{2}}\left(x_{\mu_{2}}\right)=\frac{\partial \widetilde{u}}{\partial x_{1}}\left(x_{\mu_{2}}\right)-\frac{\partial\left(\widetilde{u} \circ h_{\mu_{2}}\right)}{\partial x_{1}}\left(x_{\mu_{2}}\right)=\frac{\partial \widetilde{u}}{\partial x_{1}}\left(x_{\mu_{2}}\right) .
\end{aligned}
$$

By Hopf's lemma, we obtain

$$
-\frac{\partial \widetilde{u}}{\partial x_{1}}\left(x_{\mu_{2}}\right)<0 \text { and } \frac{\partial \widetilde{u}}{\partial x_{1}}\left(x_{\mu_{2}}\right)<0,
$$

which is a contradiction. So we have shown $v_{\mu_{2}}\left(x_{\mu_{2}}\right)<0$. Thus we have $v_{\mu_{2}}<0$ on $\Sigma_{\mu_{2}}$, which shows $\mu_{2} \in A_{2}$.

We choose an open set $G$ such that $\bar{G} \subset \Sigma_{\mu_{2}}$ and $\left|\Sigma_{\mu_{2}} \backslash \bar{G}\right| \ll 1$. We have $\max _{\bar{G}} v_{\mu_{2}}<0$. Let $0<\varepsilon \ll 1$. Then we have $\left|\Sigma_{\mu_{2}-\varepsilon} \backslash \bar{G}\right| \ll 1$ and $\max _{\bar{G}} v_{\mu_{2}-\varepsilon}<0$. Since $(2.9)$ holds with $\lambda=\mu_{2}-\varepsilon$, by the ABP inequality and the strong maximum principle, we have $v_{\mu_{2}-\varepsilon}(x)<0$ for $x \in \Sigma_{\mu_{2}-\varepsilon} \backslash \bar{G}$. Hence we have shown $v_{\mu_{2}-\varepsilon}(x)<0$ for $x \in \Sigma_{\mu_{2}-\varepsilon}$. Thus we have $\mu_{2}-\varepsilon \in A_{2}$, which is a contradiction. Therefore we obtain $\mu_{2}=0$.

Proof of Theorem 1.1. By the lemmas above, we can infer that $\widetilde{u}$ is radially symmetric and $\widetilde{u}_{r}(x)<0$ for $r=|x| \in(0,1)$. From the definition of $\widetilde{u}$, we can find $u$ is also radially symmetric and $u_{r}(x)<0$ for $r=|x| \in(0,1)$.

Proof of TheOrem 1.3. Since the arguments for the proof of Theorem 1.1 almost similarly work for that of Theorem 1.3, we prove it briefly. 
We define $\widetilde{u} \in C^{2}(D \backslash\{0\}) \cap C(\bar{D} \backslash\{0\})$ as before. For $\lambda \in(0,1)$, we define $v_{\lambda} \in C^{2}\left(\Sigma_{\lambda} \backslash\left\{x_{\lambda}\right\}\right) \cap C\left(\overline{\Sigma_{\lambda}} \backslash\left\{x_{\lambda}\right\}\right)$ by

$$
v_{\lambda}(x)=\widetilde{u}(x)-\widetilde{u}\left(h_{\lambda}(x)\right) \quad \text { for } x \in \overline{\Sigma_{\lambda}} \backslash\left\{x_{\lambda}\right\},
$$

and we define $c_{\lambda}$ by (2.7). We note that the definition of $v_{\lambda}$ is almost same as (2.6). For the sake of simplicity, we consider $v_{\lambda}\left(x_{\lambda}\right)=-\infty$ in the case $x_{\lambda} \in \overline{\Sigma_{\lambda}}$ with $\lambda \in(0,1)$. We note $c_{\lambda}$ may not belong to $L^{\infty}\left(\Sigma_{\lambda}\right)$. However, we have

$$
\sup _{r<\lambda<1} \operatorname{ess} \sup \left\{\left|c_{\lambda}(x)\right|: x \in \Sigma_{\lambda}, v_{\lambda}(x) \geq-1\right\}<\infty \quad \text { for each } r \in(0,1) .
$$

We can easily see that (2.9) holds.

We define $A_{1}$ and $\mu_{1}$ by (2.10). Since $x_{\lambda} \notin \Sigma_{\lambda}$ for $\lambda \in[\widehat{\lambda}, 1)$, we can show $\mu_{1}=\widehat{\lambda}$ by similar lines as those in the proofs of Lemmas 2.1 and 2.2. We define $A_{2}$ and $\mu_{2}$ by (2.11). By a similar proof of Lemma 2.3, we can show $A_{2} \neq \emptyset$. We will show $\mu_{2}=0$. Suppose not, i.e. $\mu_{2}>0$. Since $v_{\mu_{2}} \leq 0$ on $\overline{\Sigma_{\mu_{2}}}$, $v_{\mu_{2}} \leq-1$ in a small neighbourhood $U$ of $x_{\mu_{2}}$ with $\bar{U} \subset \Sigma_{\mu_{2}}$, and (2.9) holds for all $x \in \Sigma_{\mu_{2}} \backslash \bar{U}$, we have $v_{\mu_{2}}<0$ in $\Sigma_{\mu_{2}}$ by the strong maximum principle. Thus we have $\mu_{2} \in A_{2}$. Let $G$ be an open set such that $\bar{G} \subset \Sigma_{\mu_{2}}$ and $\left|\Sigma_{\mu_{2}} \backslash \bar{G}\right| \ll 1$. We have $\max _{x \in \bar{G}} v_{\mu_{2}}(x)<0$. Let $\lambda \in\left(0, \mu_{2}\right)$ such that $\lambda$ is sufficiently close to $\mu_{2}$. Let $V=\left\{x \in \Sigma_{\lambda}: v_{\lambda}(x)<-1\right\}$ and set $H=G \cup V$. Then we have $x_{\lambda} \in H$, $\bar{H} \subset \Sigma_{\lambda} \cup \operatorname{Int}_{\partial D}\left(\partial \Sigma_{\lambda} \cap \partial D\right), \max _{\bar{H}} v_{\lambda}<0,\left|\Sigma_{\lambda} \backslash \bar{H}\right| \ll 1$, and (2.9) holds for all $x \in \Sigma_{\lambda} \backslash \bar{H}$. By the ABP inequality and the strong maximum principle, we have $v_{\lambda}(x)<0$ for all $x \in \Sigma_{\lambda}$, which is a contradiction. Thus we have shown $\mu_{2}=0$, and we can infer that $u$ is radially symmetric and $u_{r}(x)<0$ for $r=|x| \in(0,1)$

\section{Proof of Theorem 1.4}

We will show (a). We note that each positive solution of (1.4) is of class $C^{k_{\alpha}}$, where

$$
k_{\alpha}= \begin{cases}\lfloor\alpha\rfloor+2 & \text { if } \alpha \in(0, \infty) \backslash \mathbb{N}, \\ \alpha+1 & \text { if } \alpha \text { is an odd natural number, } \\ \infty & \text { if } \alpha \text { is an even natural number. }\end{cases}
$$

Let $u$ be an $n$-mode, positive solution of (1.4) with $n \geq 1+\lceil\alpha / 2\rceil$. We choose $\widehat{n} \in \mathbb{N}$ such as $(\alpha+2) \widehat{n} \leq 2 n<2(\alpha+2) \widehat{n}$. We also choose $m \in \mathbb{N}$ such that

$$
m((\alpha+2) \widehat{n}-n) \geq n \widehat{n}, \quad m / \widehat{n} \in \mathbb{N} \text { and } m(\alpha+2)>2 n .
$$

We set $\widehat{m}=m / \widehat{n}$. We note that $\widehat{m} \geq 2$. We set $\bar{u}(r, \theta)=u\left(r^{m / n},(m / n) \theta\right)$. We note that $\bar{u}$ is $m$-mode and hence it is $\widehat{m}$-mode. From $u(r, \theta)=\bar{u}\left(r^{n / m},(n / m) \theta\right)$, 
we can easily see that

$$
\Delta \bar{u}+\left(\frac{m}{n}\right)^{2}|x|^{(m(\alpha+2)-2 n) / n} \bar{u}^{p-1}=0 \quad \text { in } D \backslash\{0\} .
$$

We will show

$$
\begin{cases}\Delta \bar{u}+\left(\frac{m}{n}\right)^{2}|x|^{(m(\alpha+2)-2 n) / n} \bar{u}^{p-1}=0 & \text { in } D, \\ \bar{u}=0 & \text { on } \partial D .\end{cases}
$$

Let $\varphi \in C_{0}^{\infty}(D)$ and let $0<\varepsilon<1$. We have

$$
\begin{aligned}
0= & \int_{\{x: \varepsilon<|x|<1\}}\left(\Delta \bar{u}+\left(\frac{m}{n}\right)^{2}|x|^{(m(\alpha+2)-2 n) / n} \bar{u}^{p-1}\right) \varphi d x \\
= & \int_{\{x: \varepsilon<|x|<1\}} \Delta \bar{u} \varphi d x+\left(\frac{m}{n}\right)^{2} \int_{\{x: \varepsilon<|x|<1\}}|x|^{(m(\alpha+2)-2 n) / n} \bar{u}^{p-1} \varphi d x \\
= & -\int_{|x|=\varepsilon} \frac{\partial \bar{u}}{\partial r} \varphi d \sigma-\int_{\{x: \varepsilon<|x|<1\}} \nabla \bar{u} \nabla \varphi d x \\
& +\left(\frac{m}{n}\right)^{2} \int_{\{x: \varepsilon<|x|<1\}}|x|^{(m(\alpha+2)-2 n) / n} \bar{u}^{p-1} \varphi d x .
\end{aligned}
$$

Noting $u \in C^{1}(\bar{D})$, and letting $\varepsilon \rightarrow 0$, we can find that $\bar{u}$ is a weak solution of (3.1). By the elliptic regularity, $\bar{u}$ is a strong solution of (3.1). Since we have

$$
\frac{m(\alpha+2)-2 n}{n}+1 \geq \widehat{m}
$$

from $m((\alpha+2) \widehat{n}-n) \geq n \widehat{n}$, we can infer $\bar{u} \in C^{\widehat{m}}(\bar{D})$. From $(\alpha+2) \widehat{n} \leq 2 n$, we can easily see

$$
r \mapsto r^{2-2 \widehat{m}+(m(\alpha+2)-2 n) / n}
$$

is nonincreasing. Noting $\bar{u}$ is $\widehat{m}$-mode and applying Theorem 1 , we obtain that $\bar{u}$ is radially symmetric, which implies that so is $u$. Hence we have shown (a).

We will show (b). We set $H_{n}=\left\{u \in H_{0}^{1}(D): u\right.$ is $n$-mode $\}$ for each $n \in \mathbb{N}$ and $H_{\infty}=\left\{u \in H_{0}^{1}(D): u\right.$ is radially symmetric $\}$. For each $\alpha \geq 0$ and $p>2$, we set

$$
S_{\alpha, p, n}=\inf _{u \in H_{n} \backslash\{0\}} R_{\alpha, p}(u) \quad \text { for each } n \in \mathbb{N} \cup\{\infty\} .
$$

For the sake of completeness, we note that $H_{1}=H_{0}^{1}(D)$ and

$$
S_{0, p, 1}=\inf _{u \in H_{0}^{1}(D) \backslash\{0\}} R_{0, p}(u) .
$$

Let $\alpha, p \in(2, \infty)$. Setting $v_{u}(|x|)=u\left(|x|^{2 /(\alpha+2)}\right)$ for each $u \in H_{\infty}$, we can find

$$
S_{\alpha, p, \infty}=\inf _{u \in H_{\infty} \backslash\{0\}}\left(\frac{\alpha+2}{2}\right)^{1+2 / p} R_{\alpha, p}\left(v_{u}\right) \geq S_{0, p, 1}\left(\frac{\alpha+2}{2}\right)^{1+2 / p} ;
$$


see [32, Theorem 4.1] and its proof. Next, let $\varphi$ be any element of $C_{0}^{\infty}(D)$. Since we can consider $\varphi \in C_{0}^{\infty}\left(\mathbb{R}^{2}\right)$ by the zero extension, we can define $\varphi_{\alpha} \in C_{0}^{\infty}(D)$ by $\varphi_{\alpha}\left(\left(x_{1}, x_{2}\right)\right)=\varphi\left(\alpha\left(x_{1}-(1-1 / \alpha)\right), \alpha x_{2}\right)$ for $\left(x_{1}, x_{2}\right) \in D$. We set $D_{1}=D$ and

$$
D_{n}=\{(r \cos \theta, r \sin \theta): 0<r<1,-\pi / n<\theta<\pi / n\} \quad \text { for } n \in \mathbb{N} \backslash\{1\} .
$$

Following the arguments in [32, Theorem 4.2], [11, Proposition 2] and [21, Lemma 1.3], we will show

$$
S_{\alpha, p, n} \leq S_{0, p, 1} n^{1-2 / p} \alpha^{4 / p}\left(\frac{\alpha}{\alpha-2}\right)^{2 \alpha / p}
$$

for each $n \in \mathbb{N}$ with $\operatorname{supp} \varphi_{\alpha} \subset D_{n}$. Let $n \in \mathbb{N}$ with $\operatorname{supp} \varphi_{\alpha} \subset D_{n}$. For the sake of completeness, we note that $\alpha \in(2, \infty)$ and $n \leq\lceil\alpha / 2\rceil$ is a sufficient condition for $\operatorname{supp} \varphi_{\alpha} \subset D_{n}$. We define $P_{n}: D \rightarrow D$ by

$$
P_{n}(r \cos \theta, r \sin \theta)=(r \cos (\theta+2 \pi / n), r \sin (\theta+2 \pi / n))
$$

for $(r, \theta) \in[0,1) \times \mathbb{R}$. We set $\widetilde{\varphi}(x)=\varphi_{\alpha}(x)+\varphi_{\alpha}\left(P_{n}(x)\right)+\ldots+\varphi_{\alpha}\left(P_{n}^{n-1}(x)\right)$ for $x \in D$. Since we have

$\int_{D}\left|\nabla \varphi_{\alpha}\right|^{2} d x=\int_{D}|\nabla \varphi|^{2} d x \quad$ and $\quad \int_{D}|x|^{\alpha}\left|\varphi_{\alpha}\right|^{p} d x \geq \alpha^{-2}\left(1-\frac{2}{\alpha}\right)^{\alpha} \int_{D}|\varphi|^{p} d x$, we obtain

$$
S_{\alpha, p, n} \leq R_{\alpha, p}(\widetilde{\varphi}) \leq \frac{n \int_{D}|\nabla \varphi|^{2} d x}{\left(n \alpha^{-2}\left(1-\frac{2}{\alpha}\right)^{\alpha} \int_{D}|\varphi|^{p} d x\right)^{2 / p}}
$$

Since $\varphi \in C_{0}^{\infty}(D)$ is arbitrary, we have shown (3.3). From (3.2) and (3.3), we can see that $n \leq n_{\alpha}$ is a sufficient condition for $S_{\alpha, p, n}<S_{\alpha, p, \infty}$.

Following [21, Lemma 1.5], we will show that if $n>1$ and $S_{\alpha, p, n}<S_{\alpha, p, \infty}$ then $S_{\alpha, p, 1}<\ldots<S_{\alpha, p, n}$. Let $n>1$ and $S_{\alpha, p, n}<S_{\alpha, p, \infty}$. We can choose $u \in H_{n} \backslash\{0\}$ such that $R_{\alpha, p}(u)=S_{\alpha, p, n}$ and $u \geq 0$. We note that $u \notin H_{\infty}$ and $u$ is a positive solution of (1.4). Let $m \in\{1, \ldots, n-1\}$. We define $v \in H_{m}$ by

$$
v(r \cos \theta, r \sin \theta)=u(r \cos (m \theta / n), r \sin (m \theta / n))
$$

for $(r, \theta) \in[0,1) \times \mathbb{R}$. Since we can see

$$
\int_{D}|x|^{\alpha}|v|^{p} d x=\int_{D}|x|^{\alpha}|u|^{p} d x
$$

and

$$
\int_{D}|\nabla v|^{2} d x=\int_{0}^{2 \pi} \int_{0}^{1}\left(\left|\frac{\partial u}{\partial r}\right|^{2}+\frac{m^{2}}{n^{2} r^{2}}\left|\frac{\partial u}{\partial \theta}\right|^{2}\right) r d r d \theta<\int_{D}|\nabla u|^{2} d x
$$


we have

$$
S_{\alpha, p, m} \leq R_{\alpha, p}(v)<R_{\alpha, p}(u)=S_{\alpha, p, n} .
$$

By a similar argument, we can conclude that $S_{\alpha, p, 1}<\ldots<S_{\alpha, p, n}$. Hence we can infer that if $S_{\alpha, p, n}<S_{\alpha, p, \infty}$, then for each $m=1, \ldots, n$, there exists a nonradial positive solution $u_{m} \in H_{m}$ of (1.4) satisfying $R_{\alpha, p}\left(u_{m}\right)=S_{\alpha, p, m}$.

We set the number in (1.6) as $\eta(\alpha, p)$. For a fixed $\alpha \in(2, \infty)$, we have $\eta(\alpha, p) \rightarrow 1+\alpha / 2$ as $p \rightarrow \infty$, which yields (i). For a fixed $p \in(2, \infty)$, we have $\eta(\alpha, p) \rightarrow \infty$ as $\alpha \rightarrow \infty$, which yields (ii). Hence, we can finish the proof of Theorem 1.4.

\section{Appendix. Proof of Theorem 1.7}

Let $a \in[-1,1] \backslash\{0\}$. We consider that $(D, g)$ is a Riemannian manifold, where the metric tensor $g$ is defined by

$$
\frac{4|a||d x|^{2}}{\left(1+a|x|^{2}\right)^{2}}
$$

For each $\lambda \in(0,1)$, let $T_{\lambda} \subset D$ be the geodesic which intersects $x_{1}$-axis orthogonally at $(\lambda, 0)$, i.e.

$$
T_{\lambda}=\left\{x \in D:\left|x-e_{\lambda}\right|=\frac{1+a \lambda^{2}}{2|a| \lambda}\right\},
$$

where

$$
e_{\lambda}=\left(-\frac{1-a \lambda^{2}}{2 a \lambda}, 0\right)
$$

For each $\lambda \in(0,1)$, we define $\Sigma_{\lambda} \subset D$ by

$$
\Sigma_{\lambda}=\left\{\begin{array}{l}
\left\{x \in D:\left|x-e_{\lambda}\right|>\frac{1+a \lambda^{2}}{2 a \lambda}\right\} \quad \text { if } a \in(0,1], \\
\left\{x \in D:\left|x-e_{\lambda}\right|<\frac{1+a \lambda^{2}}{2|a| \lambda}\right\} \quad \text { if } a \in[-1,0) .
\end{array}\right.
$$

For each $\lambda \in(0,1)$ and $x \in \Sigma_{\lambda}$, there is the reflection $h_{\lambda}(x)$ of $x$ with respect to $T_{\lambda}$ in $(D, g)$ and it is given by

$$
h_{\lambda}(x)=e_{\lambda}+\left(\frac{1+a \lambda^{2}}{2 a \lambda}\right)^{2} \frac{x-e_{\lambda}}{\left|x-e_{\lambda}\right|^{2}}=e_{\lambda}+\left(\left|e_{\lambda}\right|^{2}+\frac{1}{a}\right) \frac{x-e_{\lambda}}{\left|x-e_{\lambda}\right|^{2}} .
$$

Considering $\Sigma_{\lambda}$ as a subset of the Euclidean space $\mathbb{R}^{2}$, we can see that $h_{\lambda}$ is uniquely continuously extended to $\overline{\Sigma_{\lambda}}$. We also denote it by $h_{\lambda}$. Then $h_{\lambda}$ satisfies (A.3) for all $x \in \overline{\Sigma_{\lambda}}$. In the case of $a \neq-1,(2.3)$ holds. In the case of $a=-1$, it holds that

$$
\left|h_{\lambda}(x)\right|<|x| \text { for each } \lambda \in(0,1) \text { and } x \in \Sigma_{\lambda}
$$


and $\left|h_{\lambda}(x)\right|=1$ for each $\lambda \in(0,1)$ and $x \in \overline{\Sigma_{\lambda}} \cap \partial D$. For the proofs of (A.2), (A.3), (2.3) and (A.4), see [25], [26], [31]. We note that

$$
h_{\lambda}\left(x_{1}, 0\right)=\left(\frac{x_{1}\left(a \lambda^{2}-1\right)+2 \lambda}{2 a \lambda x_{1}+1-a \lambda^{2}}, 0\right)
$$

for $\left(x_{1}, 0\right) \in \overline{\Sigma_{\lambda}}, \lambda \in(0,1)$ and $a \in[-1,1] \backslash\{0\}$.

Now, let $n, a, f$ and $u$ be as in Theorem 1.7. For the sake of simplicity, we assume $a \neq 0$. We define $\widetilde{u} \in C^{2}(D \backslash\{0\}) \cap C(\bar{D})$ and $\widetilde{f} \in C((0,1) \times(0, \infty), \mathbb{R})$ as before. We can see that $\widetilde{u}$ and $\widetilde{f}$ satisfy (2.1) and for each $t>0$,

$$
\begin{cases}r \mapsto\left(1+a r^{2}\right)^{2} \widetilde{f}(r, t) & \text { is nonincreasing } \\ & \text { in the case of } a \in(-1,1] \backslash\{0\}, \\ r \mapsto\left(1+a r^{2}\right)^{2} \widetilde{f}(r, t) & \text { is decreasing in the case of } a=-1 .\end{cases}
$$

We set

$$
\widehat{\lambda}=\frac{1}{\sqrt{1+a}+1} \quad \text { and } \quad x_{\lambda}=\left(\frac{2 \lambda}{1-a \lambda^{2}}, 0\right) \quad \text { for } \lambda \in(0,1) .
$$

Noting (A.5), we can see that in the case of $a \in(-1,1] \backslash\{0\},(2.4)$ and (2.5) hold. In the case of $a=-1$, we can see that

$$
x_{\lambda} \in \Sigma_{\lambda} \text { and } h_{\lambda}\left(x_{\lambda}\right)=0 \text { for each } \lambda \in(0,1) .
$$

We respectively define $v_{\lambda} \in C^{2}\left(\Sigma_{\lambda} \backslash\left\{x_{\lambda}\right\}\right) \cap C\left(\overline{\Sigma_{\lambda}}\right)$ and $c_{\lambda} \in L^{\infty}\left(\Sigma_{\lambda}\right)$ by (2.6) and (2.7), and we can show (2.8). We recall that for $a \in[-1,1] \backslash\{0\}$, the Laplace-Beltrami operator on $(D, g)$ at $x \in D$ is given by

$$
\Delta_{(g, x)}=\frac{\left(1+a|x|^{2}\right)^{2}}{4|a|} \Delta .
$$

Lemma A.1. The inequality (2.9) holds.

Proof. Let $\lambda \in(0,1)$ and $x \in \Sigma_{\lambda} \backslash\left\{x_{\lambda}\right\}$. First, we will show that $h_{\lambda}:\left(\Sigma_{\lambda}, g\right)$ $\rightarrow\left(h_{\lambda}\left(\Sigma_{\lambda}\right), g\right)$ is Riemannian isometric. We set $h_{\lambda}(x)=\left(h_{\lambda, 1}(x), h_{\lambda, 2}(x)\right)$ for $x \in \Sigma_{\lambda}$. From (A.3), we have

$$
\left(\frac{\partial h_{\lambda, 1}}{\partial x_{p}}(x)\right)^{2}+\left(\frac{\partial h_{\lambda, 2}}{\partial x_{p}}(x)\right)^{2}=\left(\left|e_{\lambda}\right|^{2}+\frac{1}{a}\right)^{2} \frac{1}{\left|x-e_{\lambda}\right|^{4}} \quad \text { for } p=1,2
$$

and

$$
\frac{\partial h_{\lambda, 1}}{\partial x_{1}}(x) \frac{\partial h_{\lambda, 1}}{\partial x_{2}}(x)+\frac{\partial h_{\lambda, 2}}{\partial x_{1}}(x) \frac{\partial h_{\lambda, 2}}{\partial x_{2}}(x)=0 .
$$

Noting $2\left(x-e_{\lambda}\right) \cdot e_{\lambda}=|x|^{2}-\left|e_{\lambda}\right|^{2}-\left|x-e_{\lambda}\right|^{2}$, we can show

$$
\left(1+a\left|h_{\lambda}(x)\right|^{2}\right)\left|x-e_{\lambda}\right|^{2}=\left(1+a|x|^{2}\right)\left(\left|e_{\lambda}\right|^{2}+\frac{1}{a}\right),
$$


where $\left(x-e_{\lambda}\right) \cdot e_{\lambda}$ is the standard inner product of $\left(x-e_{\lambda}\right)$ and $e_{\lambda}$. Using these equalities and (A.1), we can easily see

$$
g_{p q}(x)=\sum_{i, j=1}^{2} g_{i j}\left(h_{\lambda}(x)\right) \frac{\partial h_{\lambda, i}}{\partial x_{p}}(x) \frac{\partial h_{\lambda, j}}{\partial x_{q}}(x) \quad \text { for } p, q=1,2 .
$$

Thus we have shown $h_{\lambda}:\left(\Sigma_{\lambda}, g\right) \rightarrow\left(h_{\lambda}\left(\Sigma_{\lambda}\right), g\right)$ is Riemannian isometric.

Let $x \in \Sigma_{\lambda}$ and set $y=h_{\lambda}(x)$. Noting $\widetilde{u} \in C^{2}(D \backslash\{0\})$, we have

$$
\Delta_{(g, y)} \widetilde{u}(y)=\Delta_{(g, x)}\left(\widetilde{u}\left(h_{\lambda}(x)\right)\right) .
$$

Then by (2.3), (A.4), (A.6), we have

$$
\begin{aligned}
0= & \Delta_{(g, y)}(\widetilde{u}(y))+\frac{\left(1+a|y|^{2}\right)^{2}}{4|a|} \widetilde{f}(|y|, \widetilde{u}(y)) \\
& -\Delta_{(g, x)} \widetilde{u}(x)-\frac{\left(1+a|x|^{2}\right)^{2}}{4|a|} \widetilde{f}(|x|, \widetilde{u}(x)) \\
= & \Delta_{(g, x)}\left(\widetilde{u}\left(h_{\lambda}(x)\right)\right)+\frac{\left(1+a\left|h_{\lambda}(x)\right|^{2}\right)^{2}}{4|a|} \widetilde{f}\left(\left|h_{\lambda}(x)\right|, \widetilde{u}\left(h_{\lambda}(x)\right)\right) \\
& -\Delta_{(g, x)} \widetilde{u}(x)-\frac{\left(1+a|x|^{2}\right)^{2}}{4|a|} \widetilde{f}(|x|, \widetilde{u}(x)) \\
\geq & -\Delta_{(g, x)} v_{\lambda}(x)+\frac{\left(1+a|x|^{2}\right)^{2}}{4|a|} \widetilde{f}\left(|x|, \widetilde{u}\left(h_{\lambda}(x)\right)\right)-\frac{\left(1+a|x|^{2}\right)^{2}}{4|a|} \widetilde{f}(|x|, \widetilde{u}(x)) \\
= & \frac{\left(1+a|x|^{2}\right)^{2}}{4|a|}\left(-\Delta v_{\lambda}(x)+c_{\lambda}(x) v_{\lambda}(x)\right) .
\end{aligned}
$$

Hence, we obtain (2.9).

Proof of Theorem 1.7. As in the case of $a=0$, we apply the moving sphere (plane) argument. First, we consider the case $a \in(-1,1] \backslash\{0\}$. Since the arguments in Section 2 work except the proof for (2.12), we give a proof for it only. Putting $r \exp (i \theta)=(r \cos \theta, r \sin \theta)$, we have

$$
\begin{aligned}
& \lim _{t \rightarrow+0} \frac{\widetilde{u}\left(h_{\mu_{2}}\left(x_{\mu_{2}}+t(1,0)\right)\right)-\widetilde{u}\left(h_{\mu_{2}}\left(x_{\mu_{2}}\right)\right)}{t} \\
& =\lim _{t \rightarrow+0} \frac{1}{t}\left[u\left(\left(\frac{t\left(1-a \mu_{2}^{2}\right)^{2}}{2 a \mu_{2}\left(2 \mu_{2}-\left(1-a \mu_{2}^{2}\right) t\right)+\left(1-a \mu_{2}^{2}\right)^{2}}\right)^{1 / n} \exp \left(\frac{i \pi}{n}\right)\right)-u(0,0)\right] \\
& \lim _{t \rightarrow+0} \frac{\widetilde{u}\left(h_{\mu_{2}}\left(x_{\mu_{2}}-t(1,0)\right)\right)-\widetilde{u}\left(h_{\mu_{2}}\left(x_{\mu_{2}}\right)\right)}{t} \\
& =\lim _{t \rightarrow+0} \frac{1}{t}\left[u\left(\left(\frac{t\left(1-a \mu_{2}^{2}\right)^{2}}{2 a \mu_{2}\left(2 \mu_{2}-\left(1-a \mu_{2}^{2}\right) t\right)+\left(1-a \mu_{2}^{2}\right)^{2}}\right)^{1 / n}, 0\right)-u(0,0)\right]=0,
\end{aligned}
$$

which yield (2.12). We note that these calculations are also valid for $a=-1$. 
Next, we consider the case $a=-1$. Since we do not need to define $A_{1}$ and $\mu_{1}$ in this case, we start the moving sphere arguments in Section 2 by setting $A_{2}$ and $\mu_{2}$. We can easily see $A_{2} \neq \emptyset$. Since the arguments in Section 2 also work except the proof for the first paragraph of Lemma 2.5, we give a proof for it only. That is, assuming $\mu_{2} \in(0, \widehat{\lambda})$, we will show $v_{\mu_{2}}<0$ on $\Sigma_{\mu_{2}} \backslash\left\{x_{\mu_{2}}\right\}$. By (A.4), (2.9) with $\lambda=\mu_{2}$ and the strong maximum principle, we have $v_{\mu_{2}}=0$ on $\Sigma_{\mu_{2}}$ or $v_{\mu_{2}}<0$ on $\Sigma_{\mu_{2}} \backslash\left\{x_{\mu_{2}}\right\}$. If $v_{\mu_{2}}=0$ on $\Sigma_{\mu_{2}}$, from the calculation in the proof of Lemma (A.1), we have

$$
\left(1-|x|^{2}\right)^{2} \widetilde{f}(|x|, \widetilde{u}(x))=\left(1-\left|h_{\mu_{2}}(x)\right|^{2}\right)^{2} \widetilde{f}\left(\left|h_{\mu_{2}}(x)\right|, \widetilde{u}(x)\right)
$$

for $x \in \Sigma_{\mu_{2}} \backslash\left\{x_{\mu_{2}}\right\}$, which contradicts (A.6). Thus we have shown $v_{\mu_{2}}<0$ on $\Sigma_{\mu_{2}} \backslash\left\{x_{\mu_{2}}\right\}$.

Proof of Corollary 1.9. Let $m \in \mathbb{N}$ with $m>n$. Since

$$
\begin{aligned}
\frac{d}{d r}\left(\frac{r^{-m}+a r^{m}}{r^{-n}+a r^{n}}\right) & =\frac{(m-n)\left(a^{2} r^{m+n}-r^{-m-n}\right)+(m+n)\left(a r^{m-n}-a r^{-m+n}\right)}{r\left(r^{-n}+a r^{n}\right)^{2}} \\
& \equiv \frac{\gamma(r)}{r\left(r^{-n}+a r^{n}\right)^{2}}, \\
\frac{d}{d r} \gamma(r) & =\frac{m^{2}-n^{2}}{r}\left(r^{-m}+a r^{m}\right)\left(r^{-n}+a r^{n}\right),
\end{aligned}
$$

and $\gamma(1) \leq 0$, the function $r \mapsto\left(1+a r^{2 m}\right)^{2} r^{2-2 m} /\left(\left(1+a r^{2 n}\right)^{2} r^{2-2 n}\right)$ is decreasing in $(0,1)$. From $f \geq 0$, we can find that for each $m \in \mathbb{N}$ with $m \geq n$, (a) in Theorem 1.7 holds for $m$ instead of $n$. Now, assume that $u$ is $m$-mode with $n \leq m \leq k$. Then from Theorem 1.7 , we can see that $u$ is radially symmetric.

\section{REFERENCES}

[1] L. Almeida, Y. Ge and G. Orlandi, Some connections between symmetry results for semilinear PDE in real and hyperbolic spaces, J. Math. Anal. Appl. 311 (2005), 626-634.

[2] T. Bartsch, T. Weth and M. Willem, Partial symmetry of least energy nodal solutions to some variational problems, J. Anal. Math. 96 (2005), 1-18.

[3] E. Berchio, F. Gazzola and T. Weth, Radial symmetry of positive solutions to nonlinear polyharmonic Dirichlet problems, J. Reine Angew. Math. 620 (2008), 165183.

[4] H. Berestycki and L. Nirenberg, Monotonicity, symmetry and antisymmetry of solutions of semilinear elliptic equations, J. Geom. Phys. 5 (1988), 237-275.

[5] On the method of moving planes and the sliding method, Bol. Soc. Brasil. Mat. (N.S.) 22 (1991), 1-37.

[6] H. Berestycki, L.A. Caffarelli and L. Nirenberg, Monotonicity for elliptic equations in unbounded Lipschitz domains, Comm. Pure Appl. Math. 50 (1997), 1089-1111.

[7] G. Bianchi, Non-existence of positive solutions to semilinear elliptic equations on $\mathbb{R}^{n}$ or $\mathbb{R}_{+}^{n}$ through the method of moving planes, Comm. Partial Differential Equations 22 (1997), 1671-1690. 
[8] H. BREzIs, Symmetry in nonlinear PDE's, Differential equations: La Pietra 1996 (Florence), Proc. Sympos. Pure Math., vol. 65, Amer. Math. Soc., Providence, RI, 1999, pp. $1-12$.

[9] F. BRock AND J. PRAJAPAT, Some new symmetry results for elliptic problems on the sphere and in Euclidean space, Rend. Circ. Mat. Palermo (2) 49 (2000), 445-462.

[10] W. Chen And C. Li, Moving planes, moving spheres, and a priori estimates, J. Differential Equations 195 (2003), 1-13.

[11] C.V. Coffman, A nonlinear boundary value problem with many positive solutions, J. Differential Equations 54 (1984), 429-437.

[12] B. Gidas, W.M. Ni And L. Nirenberg, Symmetry and related properties via the maximum principle, Comm. Math. Phys. 68 (1979), 209-243.

[13] Symmetry of positive solutions of nonlinear elliptic equations in $\mathbb{R}^{n}$, Mathematical Analysis and Applications, Part A, Adv. in Math. Suppl. Stud., vol. 7, Academic Press, New York, 1981, pp. 369-402.

[14] D. Gilbarg And N.S. Trudinger, Elliptic partial differential equations of second order, Classics in Mathematics, Reprint of the 1998 edition, Springer-Verlag, Berlin, 2001, pp. $x i v+517$.

[15] F. Gladiali, F. Pacella and T. Weth, Symmetry and nonexistence of low Morse index solutions in unbounded domains, J. Math. Pures Appl. (9) 93 (2010), 536-558.

[16] R. KAJIKIYA, Non-radial least energy solutions of the generalized Hénon equation, J. Differential Equations 252 (2012), 1987-2003.

[17] S. Kumaresan And J. PRAJAPAT, Serrin's result for hyperbolic space and sphere, Duke Math. J. 91 (1998), 17-28.

[18] A.C. Lazer And P.J. MCKenna, A symmetry theorem and applications to nonlinear partial differential equations, J. Differential Equations 72 (1988), 95-106.

[19] C. LI, Monotonicity and symmetry of solutions of fully nonlinear elliptic equations on bounded domains, Comm. Partial Differential Equations 16 (1991), 491-526.

[20] _ Monotonicity and symmetry of solutions of fully nonlinear elliptic equations on unbounded domains, Comm. Partial Differential Equations 16 (1991), 585-615.

[21] Y.Y. Li, Existence of many positive solutions of semilinear elliptic equations on annulus, J. Differential Equations 83 (1990), 348-367.

[22] Y. LI, On the positive solutions of the Matukuma equation, Duke Math. J. 70 (1993), $575-589$.

[23] Y. Li AND W.-M. Ni, Radial symmetry of positive solutions of nonlinear elliptic equations in $\mathbb{R}^{n}$, Comm. Partial Differential Equations 18 (1993), 1043-1054.

[24] Y. NAIto, Radial symmetry of positive solutions for semilinear elliptic equations in $\mathbb{R}^{n}$, J. Korean Math. Soc. 37 (2000), 751-761.

[25] Y. Naito, T. Nishimoto And T. Suzuki, Radial symmetry of positive solutions for semilinear elliptic equations in a disc, Hiroshima Math. J. 26 (1996), 531-545.

[26] Y. NAito AND T. Suzuki, Radial symmetry of positive solutions for semilinear elliptic equations on the unit ball in $\mathbb{R}^{n}$, Funkcial. Ekvac. 41 (1998), 215-234.

[27] _ A note of the moving sphere method, Pacific J. Math. 189 (1999), 107-115.

[28] F. Pacella AND T. Weth, Symmetry of solutions to semilinear elliptic equations via Morse index, Proc. Amer. Math. Soc. 135 (2007), 1753-1762.

[29] P. PADIlla, Symmetry properties of positive solutions of elliptic equations on symmetric domains, Appl. Anal. 64 (1997), 153-169.

[30] J. SERrin, A symmetry problem in potential theory, Arch. Rational Mech. Anal. 43 (1971), 304-318. 
[31] N. Shioji And K. Watanabe, Radial symmetry of positive solutions for semilinear elliptic equations in the unit ball via elliptic and hyperbolic geometry, J. Differential Equations 252 (2012), 1392-1402.

[32] S. Smets, M. Willem AND J. Su, Non-radial ground states for the Hénon equation, Commun. Contemp. Math. 4 (2002), 467-480.

[33] J. VAn SChaftingen And M. WILlem, Symmetry of solutions of semilinear elliptic problems, J. Eur. Math. Soc. 10 (2008), 439-456.

NAOKI SHIOJI

Department of Mathematics

Faculty of Engineering

Yokohama National University

Tokiwadai, Hodogaya-ku

Yokohama 240-8501, JAPAN

E-mail address: shioji@ynu.ac.jp

Kohtaro Watanabe

Department of Computer Science

National Defense Academy

1-10-20 Hashirimizu

Yokosuka 239-8686, JAPAN

E-mail address: wata@nda.ac.jp 